Revista Ingeniería y Región. 2015;14(2):87-97

\title{
El transporte sostenible y su papel en el desarrollo del medio ambiente urbano
}

\section{Sustainable transport and its role in the development of urban environment}

\author{
Julián Rodrigo Quintero González y Laura Estefanía Quintero González²
}

\begin{abstract}
Resumen
Este artículo presenta un análisis acerca de la problemática actual de la movilidad urbana representada en dificultades puntuales como la congestión, falta de accesibilidad al servicio de transporte, ocupación del espacio público e impactos en el medio ambiente, entre otros; problemas que son originados por algunos de los sistemas de transporte más utilizados en las grandes ciudades. Son mencionados además los modelos de transporte actuales como el metro y el Bus Rapid Transit (BRT), que si bien colaboran en el desarrollo del medio ambiente urbano, existen sistemas de transporte alternativo que maximizan dicha labor. Por esto mismo, el objetivo de este documento es mostrar algunas de las tendencias de sistemas de transporte alternativo y su papel en la solución de las problemáticas anteriormente mencionadas; sus principales características y sus ventajas a nivel de movilidad, a nivel social y ambiental. Así mismo, las directrices bajo las cuales es ideal la planeación e implementación de mecanismos de transporte para el desarrollo sostenible urbano son descritas de manera general.
\end{abstract}

Palabras clave: Sistemas de transporte alternativos; movilidad urbana sostenible; transporte y medio ambiente.

\begin{abstract}
This paper presents an analysis of the current problems of urban mobility represented on specific problems such as congestion, lack of accessibility to the transportation, occupation of public space and impacts on the environment, among others; problems that are caused by some of the most widely used transport systems in large cities. In addition, the current transport models such as the metro and Bus Rapid Transit (BRT) are mentioned, that while collaborating on the development of the urban environment, alternative transportation systems exist to maximize these efforts. For this reason, the aim of this paper is to show some trends of alternative transportation systems and their role in solving the above problems; its main characteristics and its advantages in terms of mobility, social and environmental level. Also, the guidelines under which is ideal planning and implementation of transport mechanisms for sustainable urban development are described in general.
\end{abstract}

Key words: Alternative transportation systems; sustainable urban mobility; transport and environment.

1 Magíster en Ingeniería Ambiental, Universidad Pedagógica y Tecnológica de Colombia, Tunja, Avenida Central del Norte, julian.quintero@uptc.edu.co

2 Ingeniera Ambiental, Universidad Pedagógica y Tecnológica de Colombia, Tunja, Avenida Central del Norte, lauraestefania.quintero@uptc.edu.co

Recibido: 3 julio 2015 - Aceptado: 16 octubre 2015 


\section{Introducción}

En todo el mundo las necesidades de transporte derivadas del crecimiento acelerado de las grandes ciudades, que a su vez ha traído consigo el aumento de problemas de movilidad urbana (Banco de Desarrollo de América Latina CAF, 2011), ponen en evidencia la necesidad de buscar e implementar nuevas medidas que permitan garantizar la movilidad de los ciudadanos a la vez que se contrarresten problemas de tráfico tales como la congestión, accidentalidad, baja calidad de los servicios de transporte, falta de accesibilidad al servicio de transporte, ocupación del espacio público e impactos en el medio ambiente (Transportation Research Board, 2005). Aunque el estudio de los diferentes modos y medios de transporte y el comportamiento del tráfico vehicular se ha perfeccionado en las últimas décadas, inicialmente éste se enfocó principalmente en el análisis de la demanda de viajes, la valoración de la calidad del servicio, la determinación de los niveles de servicio de calles y carreteras y el estudio de algunas externalidades como la tarifa, tendencia que aún se mantiene en países en vía de desarrollo, algunos de éstos en Latinoamérica (Mesquita et al., 2008).

Soluciones tradicionales como el aumento del parque automotor para satisfacer la demanda de viajes, el cambio de un determinado número de vehículos de servicio público por un número menor de vehículos de mayores capacidades; que en algunos casos ocupan mayor espacio o contaminan más, estudios extensos de orígenes y destinos y de externalidades del transporte como el diseño de tarifas sin aparentes resultados favorables, han estado dirigidos más a establecer medidas que satisfagan en forma eficaz el problema del transporte. No obstante, aspectos considerados como de primer orden como el uso adecuado del espacio público, la accesibilidad al medio físico y al propio sistema de transporte y sobretodo la preservación del medio ambiente y la salud, y que hasta hace algunos años habían pasado a ocupar un segundo lugar dentro de las políticas de desarrollo de las ciudades (Fedesarrollo, 2013), ahora son considerados pilares fundamentales en la búsqueda de una verdadera sostenibilidad del transporte coherente con las directrices de las nuevas políticas en materia de medio ambiente.

Las múltiples problemáticas generadas en torno a la actividad del transporte han impulsado el avance de nuevas y más eficientes estrategias orientadas al mejoramiento y preservación del medio ambiente en áreas urbanas y periféricas en las ciudades para lo cual, los gobiernos en todo el mundo, a través de las administraciones municipales en las grandes ciudades y ciudades intermedias han fomentado el desarrollo de nuevos proyectos de movilidad urbana basados en la implementación de sistemas de transporte alternativo y sostenible tales como el tranvía, cables, bicicleta y peatonal, los cuales salvaguardan las premisas de movilidad sostenible, manejo de la demanda, uso del suelo, preservación del medio ambiente y la salud pública (Litman y Burwell, 2006).

Otras estrategias puestas en marcha y que han arrojado muy buenos resultados en países de Europa y Asia incluyen avances tecnológicos en los vehículos particulares, de transporte público y transporte masivo, el uso de combustibles alternativos y la creación de redes amplias para su distribución (World Economic Forum, 2012), la implementación de mejores infraestructuras para el desarrollo de modos de transporte no motorizado pero sobretodo, el estudio detallado de las verdaderas necesidades de los usuarios no bajo un esquema de eficacia sino dentro del marco del transporte y movilidad urbana sostenibles eficientes lejos de los bocetos tradicionales. Atendiendo a lo anterior, se desarrolló una investigación cuyos resultados se muestran en el presente artículo, con el objetivo de exponer los principales aspectos relacionados con las características de los actuales modelos de movilidad urbana desde la perspectiva del tráfico, sociedad, cultura, economía, política, ciencia y tecnología, además de las tendencias actuales en relación al transporte sostenible y su importancia como elemento del medio ambiente urbano.

\section{Movilidad urbana y problemática}

\subsection{Tráfico y movilidad urbana}

Alcântara (2010) explica que el modelo general del desarrollo urbano está dado por la configuración de las ciudades en los lugares en que las personas realizan sus actividades cotidianas, algunas de las cuales se desarrollan fuera de sus viviendas y para las que requieren el uso de diversos medios para desplazarse tales como la caminata, el uso de bicicleta, automóviles, autobuses, motocicletas, ferrocarriles y el metro. Estudios recientes señalan que los problemas de movilidad urbana son el resultado de la rápida urbanización y tiene múltiples efectos en las economías urbanas para lo cual podría definirse la congestión urbana como el exceso de demanda de viajes sobre su oferta (Alam y Ahmed, 2013), en palabras de Rao y Rao (2012) la congestión del tráfico urbano consiste 
en que mucha gente quiere moverse al mismo tiempo y existen demasiados vehículos para el espacio vial limitado (Figura 1).

De acuerdo con Arboleda et al. (2012) los eventuales problemas de movilidad en las ciudades también pueden ser ocasionados por la incapacidad de una malla vial para soportar flujos vehiculares sujetos a crecimientos acelerados en ciudades con pocos recursos para el desarrollo de infraestructura vial, razón por la cual el incremento de la red vial no es una solución inmediata al problema de movilidad en las ciudades. De otra parte se indica que la accidentalidad es uno de los problemas que tiene mayor afectación en el tráfico vehicular, así como el hecho que las medidas tomadas para controlar la congestión como la restricción vehicular sólo producen una distribución de la carga en el corto plazo pero tampoco solucionan el problema en forma efectiva.

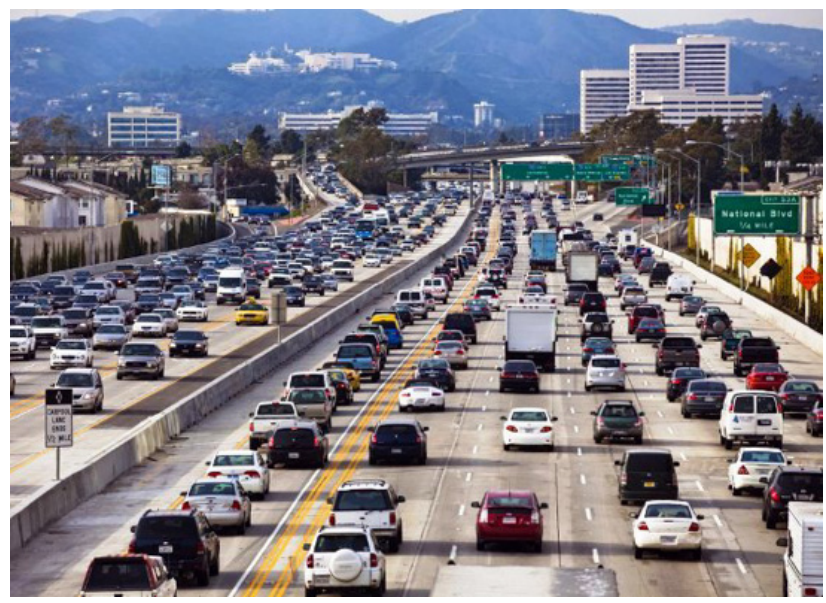

Figura 1. Congestión en autopista en Los Ángeles, Estados Unidos. Fuente: http://img.diariodelviajero.com/2010/07/ lax.jpg

\subsection{Perspectiva social, cultural, económica y política}

El actual modelo de movilidad caracterizado por el aumento de las distancias medias recorridas, los cambios en los motivos de viaje y las modificaciones en la localización de las actividades productivas, trae consigo cambios en las características sociales y económicas de las ciudades. Esto se puede apreciar en las crecientes tasas de motorización derivadas del aumento de los ingresos per cápita, para lo cual, el incremento de compra y uso del vehículo particular ha generado una disminución en el uso de transporte público colectivo urbano (Lizárraga, 2006). Desde la perspectiva social y cultural, la necesidad de desplazamiento ha originado una interacción parcial entre individuos, trayendo consigo relaciones sociales dispersas y trasformaciones en las personas sujetas a una vida social en las grandes ciudades, caracterizadas por actitudes menos solidarias, de superficialidad e individualidad, que trascienden en la existencia de relaciones sociales conflictivas entre clases sociales, y derivan en un modelo de gestión de la movilidad desigual en cuanto a la accesibilidad al medio físico y al transporte (Lange, 2011).

Desde el punto de vista económico, las externalidades negativas siguen siendo un factor problemático, los costos externos del transporte; representados principalmente por aquellos derivados de los accidentes de tránsito, el ruido, la contaminación atmosférica, los riesgos de cambio climático, los costos para la naturaleza, el paisaje y áreas urbanas y la congestión, plantean un claro problema de sostenibilidad ambiental y energética, que a la vez enmarcan la insostenibilidad urbana de los modelos de movilidad convencionales (Lizárraga, 2006). Desde la perspectiva del Estado y las políticas públicas de movilidad está claro que esta debe considerarse como un derecho que, garantizado, contribuye al mejoramiento de la calidad de vida de los ciudadanos, con o sin limitaciones, en un marco definido por la igualdad, el ambiente sano y el desarrollo sostenible (Dangond et al., 2011). Sin embargo, la ampliación de las capacidades de avenidas y autopistas, que potencian la congestión futura, a la vez que se pretende aventajar al transporte masivo, podrían indicar la falta de uniformidad de las políticas públicas de movilidad (Lupano y Sánchez, 2009). Esta inadecuada planeación y disfuncional aplicación de las políticas creadas por el Estado, y en ocasiones implementadas por él mismo, han ocasionado la fragmentación de las ciudades, la carencia de articulación social, económica y funcional, y la desigualdad de los servicios de transporte en áreas urbanas ahondando los problemas de movilidad urbana (Dangond et al., 2011).

\subsection{Perspectiva científica y tecnológica}

Considerando el conocimiento de que toda actividad humana genera algún tipo de impacto y/o contaminación; tanto por el uso desmedido de los recursos naturales como por los impactos negativos generados sobre éstos, la inclusión de tecnologías tiene un objetivo claro que es la explotación de los sistemas de transporte urbanos bajo la premisa de la efectividad en la movilidad y el aprovechamiento de los recursos naturales, garantizando su conservación, restauración o sustitución. No obstante, el deterioro ambiental eleva los costos de producción y la pérdida de recursos, puesto que las actividades de los sectores productivos como el 
transporte tienen externalidades que se deben asumir y que tienen importantes repercusiones sociales y económicas, lo cual a su vez, implica la disminución de las posibilidades de acceso a los recursos (Lara y Moreno, 2014). Esto limita enormemente el gasto en investigación científica así como desarrollos tecnológicos en materia de movilidad urbana sostenible, lo que por supuesto responde a una simbiosis directa con los factores político, económico, social y cultural anteriormente descritos, lo que finalmente conlleva a un círculo vicioso de aumento de la contaminación y deterioro ambiental así como problemas de crecimiento de la pobreza y de exclusión social.

\section{Los sistemas de transporte en las ciudades}

\subsection{Modos y medios de transporte urbano}

Se puede definir una clasificación de modos de transporte que se desarrollan en las ciudades, discriminándolos en tres grupos; terrestre, aéreo y acuático, los cuales a la vez están compuestos por los denominados "medios" que permiten obtener una mejor clasificación de las diferentes formas en que se desarrolla el transporte (Tabla 1).

En forma similar a la clasificación de acuerdo al medio, también se pueden definir diversos tipos de transporte atendiendo a la naturaleza de las actividades que desarrolla, en éste aspecto se podrían distinguir: el transporte público, el transporte privado, el de pasajeros y el de carga; éste último podría ser general, de alimentos, de animales y de mercancías. Atendiendo a su interacción con el medio urbano, los sistemas de transporte en las ciudades guardan una relación profunda con los problemas propios de algunos sistemas de transporte urbano, entre éstos el consumo energético de fuentes no renovables, el incremento en los niveles de contaminación atmosférica, emisiones de gases contaminantes y ruido, y el desarrollo de asentamientos suburbanos en zonas periféricas, que generan más cantidades de viajes y a la vez mayores problemas de movilidad (Colmenares, 2007).

\subsection{Mirada a los modelos de transporte actuales}

En la actualidad, en casi todas las grandes ciudades del mundo, el modelo de transporte urbano que se maneja está constituido por dos clases principales de transporte público, el colectivo y el masivo. La primera clase; denominado transporte público colectivo, se caracteriza por estar compuesto por buses que se desplazan por vías compartidas con otros tipos de vehículos motorizados y en algunos casos no motorizados, como la bicicleta, lo que implica el aumento del problema de la congestión en vías, el incremento de los tiempos de viaje, mayor número de paradas para el ascenso y descenso de pasajeros, mayores tiempos de espera y efectos contaminantes más nocivos que el transporte masivo (Banco Mundial, 2002). En relación al transporte masivo representado por el metro y el Bus Rapid Transit (BRT), se ha proporcionado una solución eficiente al problema de movilidad en las ciudades; el metro, que se alimenta de energía eléctrica, ofrece altos rendimientos en referencia a la capacidad de pasajeros transportados y beneficios al medio ambiente por no generar emisiones de gases contaminantes; el BRT por su parte, emplea buses de alta capacidad que en algunos casos utilizan combustibles limpios, los cuales se desplazan por vías exclusivas con estaciones fijas y accesibles, conjunto que permite disminuir impactos en el medio ambiente a la vez que se disminuyen los tiempos de recorrido y las demoras ocasionadas por el tráfico; lo cual se traduce en una reducción de tiempos de viajes para los usuarios en lo que se considera un modo de transporte eficiente (Figura 2). En los últimos años el metro y el BRT han sido los medios de transporte público predominantes, especialmente en países en desarrollo que ven en éstos sistemas ventajas en términos de capacidad de transporte de pasajeros con costos comparativamente bajos (Navas, 2008).

Tabla 1. Modos y medios de transporte.

\begin{tabular}{l|l|l}
\hline Modo & Medio & Unidades Transportadoras \\
\hline \multirow{3}{*}{ TERRESTRE } & Carretero & $\begin{array}{l}\text { Automóvil, Bus, Bus Metropolitano, Camión, Motocicleta, } \\
\text { Bicicleta, Peatonal. }\end{array}$ \\
\cline { 2 - 3 } & Ferroviario & Tren, Metro, Tranvía, Tren de Alta Velocidad. \\
\cline { 2 - 3 } & Cables & Teleférico, Funicular, Ferrocarril Funicular, Telecabina. \\
\hline \multirow{2}{*}{ AÉREO } & Aéreo Aerodinos & Aviones, Helicópteros. \\
\cline { 2 - 3 } & Aéreo Aerostatos & Globo Aerostático, Dirigible. \\
\hline ACUÁTICO & Fluvial & Transbordador o Ferry. \\
\hline
\end{tabular}

Fuente: Los autores. 


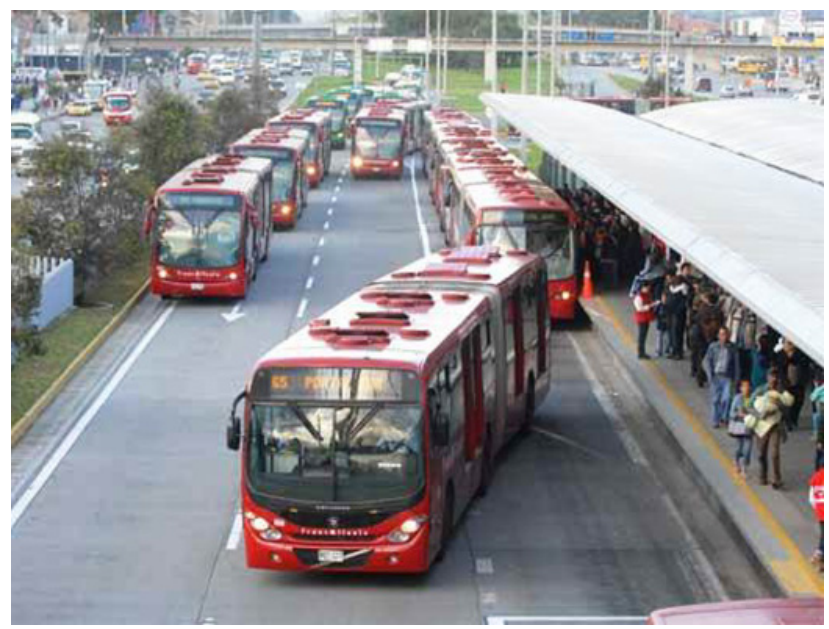

Figura 2. Sistema Integrado de Transporte Transmilenio, Bogotá, Colombia. Fuente: http://st.elespectador.co/files/ images/3be21a17bb338345c3a875fab7b527c6.jpg

\section{Transporte sostenible alternativo y medio ambiente urbano}

\subsection{Inicios del transporte alternativo}

Colmenares (2007) explica que las tendencias mundiales apuntan al tratamiento del problema de movilidad en las ciudades en tres frentes que son la equidad social, el equilibrio del medio ambiente y el valor económico, así la tendencia mundial apunta al desarrollo de sistemas que sean capaces de transportar un gran número de personas tales como los metros, metros ligeros, trolebuses, autobuses y recientemente los sistemas de transporte por cables. Sistemas como el metro ligero resultan más rentables desde el punto de vista del consumo de energía, y eventualmente más eficientes en la medida que se alimenten de energías renovables. En general la premisa en el marco global es la de diseñar sistemas que cumplan los requerimientos mínimos respecto a la calidad y cantidad del servicio prestado, velocidad, seguridad, índices de contaminación ambiental y consumo energético, a la vez que se garantizan las interacciones entre las actividades económicas y sociales.

En el marco europeo el rumbo se ha fijado en un esquema de transporte urbano limpio basado en la generación de grandes proyectos de eficiencia energética a través de flotas de vehículos limpios equipados con motores que usan biocombustibles y gas natural, complementado por estrategias como la integración de redes, mejoramiento de la accesibilidad, nuevas formas de utilización del vehículo particular y la integración de los sistemas de gestión. En Latinoamérica el esquema manejado en los últimos años se ha enfocado en la reestructuración del espacio urbano, el control del crecimiento del uso del vehículo particular y la implementación de sistemas de troncales de transporte masivo urbano y suburbano en casos como el de Caracas (Venezuela), Santiago de Chile (Chile) y Bogotá Distrito Capital (Colombia).

\subsection{Camino hacia el transporte sostenible; mecanismos}

En el marco internacional actual, el instrumento de planificación de movilidad urbana más utilizado, especialmente en Europa, es el Plan de Movilidad Urbana Sostenible (PMUS), el cual, debe incluir como mínimo un análisis de la situación actual, diagnóstico, definición de objetivos, definición de un escenario de movilidad futuro deseado, propuesta de medidas a tomar, selección de medidas y programa de actuación, que además está constituido por cuatro grupos principales de estrategias así (González, 2011):

1. La reducción de la necesidad de transporte; planificación urbana, reducción de emisiones, reducción de transporte motorizado.

2. La potencialización del cambio modal; uso de modos de transporte alternativo al vehículo particular.

3. Desarrollo de sistemas de transporte limpios; vehículos con bajas emisiones y uso de combustibles alternativos.

4. Mejoramiento de la eficiencia del transporte; reducción de impactos ambientales a través del control de los flujos vehiculares, la organización y gestión del tráfico.

En Colombia la legislación vigente, específicamente establecida por la Ley 1083 de 2006 del Ministerio de Ambiente y Desarrollo Sostenible (Ministerio de Ambiente, Vivienda y Desarrollo Territorial, 2006), es clara al obligar a aquellos municipios que cuenten con más de 100.000 habitantes, a desarrollar Planes de Ordenamiento Territorial (POT) dentro de los cuales se formulen y adopte Planes de Movilidad Urbana "con el fin de dar prelación a la movilización en modos alternativos de transporte, entendiendo por éstos el desplazamiento peatonal, en bicicleta o en otros medios no contaminantes, así como los sistemas de transporte público que funcionen con combustibles limpios" (Artículo 1, Ley 1083 de 2006). Adicionalmente, la ley mencionada establece que los Planes de Movilidad deberán seguir los siguientes lineamientos: 1. Identificar los componentes relacionados con la movilidad, 
incluidos en el Plan de Ordenamiento Territorial 2. Articular los sistemas de movilidad con la estructura urbana propuesta en el Plan de Ordenamiento Territorial 3. Reorganizar las rutas de transporte público y tráfico 4. Crear zonas sin tráfico vehicular 5. Crear zonas de emisiones bajas y 6. Incorporar un Plan Maestro de Parqueaderos.

De ésta manera, la inclusión de nuevas políticas como mecanismo para el fomento del desarrollo del transporte basadas en la planificación territorial integrada e integral, la coordinación de las administraciones, la participación ciudadana, la reducción de la necesidad de movilizarse, el aseguramiento de la sustentabilidad en el largo plazo, la participación privada, la eficiencia de la gestión de la infraestructura y la elaboración de normas más estrictas en materia de emisiones, inmersas dentro de un modelo de normativa, capacidad, organización y gestión de procesos (Holuigue, 2011), se muestran como la principal herramienta; $y$ que debe seguirse empleando, en el camino a un esquema de transporte sostenible urbano orientado hacia la Calidad del Medio Ambiente, el Desarrollo Económico y la Equidad Social (Environmental Protection Agency, 2011).

\subsection{Tendencias de los sistemas de transporte alternativo}

En relación al uso de sistemas de transporte masivo alternativo se debe resaltar la rápida evolución y beneficios del tranvía en países como Francia, en donde el tranvía interactúa con los espacios urbanos de diversas maneras dado que se desplaza en forma abierta por la ciudad y es visible desde la calle (Figura 3). Los arquitectos paisajistas utilizan esta visibilidad como una oportunidad para la renovación de calles entorno al sistema tranviario, a la vez que restauran el nivel de confort y la calidad de vida de los ciudadanos en centros urbanos, que anteriormente se veían reducidos por la presencia de los vehículos. Así, el tranvía proporciona una mejora inmediata del medio ambiente a través de la reducción de la contaminación del aire, disminución de niveles de ruido del tráfico, revegetalización de zonas contiguas, siembra de árboles y en general una transformación benéfica del paisaje urbano (Ministry for Ecology, Sustainable Development and Energy, 2012).

Estimaciones hechas en Londres (Inglaterra) señalan que el tranvía puede transportar a 320 pasajeros en forma ágil y segura, número mucho mayor al transportado por un servicio regular de buses (London Assembly Transport Committee, 2004). En Francia y el Reino Unido el tranvía ha logrado diversas ventajas

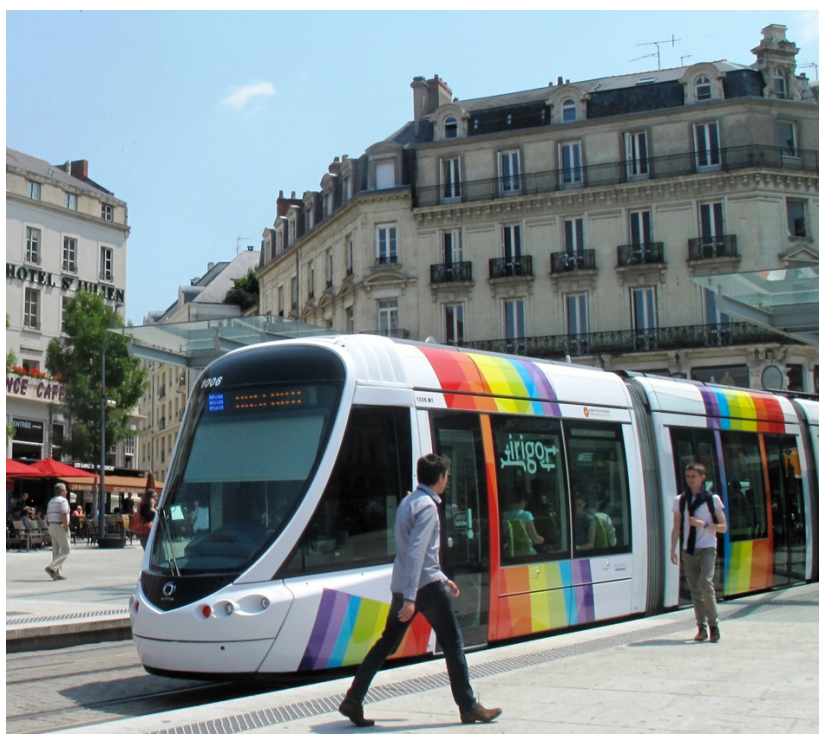

Figura 3. Tranvía Irigo Ciudad de Angers, Francia. Fuente: http://www.rue-avenir.ch/fileadmin/user_ upload/resources/Angers-pl-Ralliement - tram.jpg

como una excelente movilidad y confort para todos los pasajeros, seguridad y confiabilidad, nuevas configuraciones para diversas capacidades, eliminación de catenarias, consumo energético reducido para costos de ciclo de vida optimizados, y compatibilidad con el medio ambiente (Alstom, 2014; Griffiths, 2012).

De otra parte, y de acuerdo con lo expuesto por Orro et al. (2003) los sistemas de transporte por cables ofrecen múltiples ventajas, para lo cual distinguen las siguientes:

1. Facilidad para superar las irregularidades del terreno, así como pendientes longitudinales y transversales, si se hace la elección del sistema adecuado.

2. Considerando el mismo personal en el servicio, una significativa mayor capacidad horaria frente al transporte carretero.

3. Automatización del sistema de transporte y regularidad del servicio.

4. Desde el punto de vista económico se logra una rápida recuperación del capital invertido, el cual es bajo en la etapa de instalación.

Su versatilidad basada en el diseño y construcción de sistemas novedosos y combinaciones de éstos ha permitido su uso en áreas urbanas (funiculares en ciudades como Lyon, Barcelona, Innsbruck y El Havre, entre 
otros), incluso con fines turísticos (Barcelona, Colonia y Lisboa), además de contar con casos ejemplares de inclusión como sistema de transporte público en todo el mundo (Medellín, Caracas, Río de Janeiro, Portland, Nueva York, Argel) (Certu, 2011). En referencia a exclusión social como uno de los problemas más significativos de los últimos tiempos (Jirón, 2012), ciertamente el más importante aporte del trasporte por cables es la accesibilidad, el derecho a la ciudad y la justicia social, desarrolladas mediante la inclusión social y espacial de la periferia, y la homogenización de sociedad a través del mejoramiento de la movilidad (Leibler y Brand, 2012). Además es considerado un modo de transporte limpio, disminuyendo directamente las emisiones de dióxido de carbono, éste es el caso de los teleféricos de Caracas en Venezuela (Figura 4), pionero en la década del 2000 (Landrin, 2014) y de Medellín, Colombia, donde el sistema denominado Metrocable se muestra como una experiencia innovadora, dado que se aplicó una tecnología más asociada a la industria turística a sectores de bajos ingresos, constituyéndose en una respuesta económica, rápida y visible a los problemas de transporte urbano y de movilidad en la ciudad (Leibler y Brand, 2012).

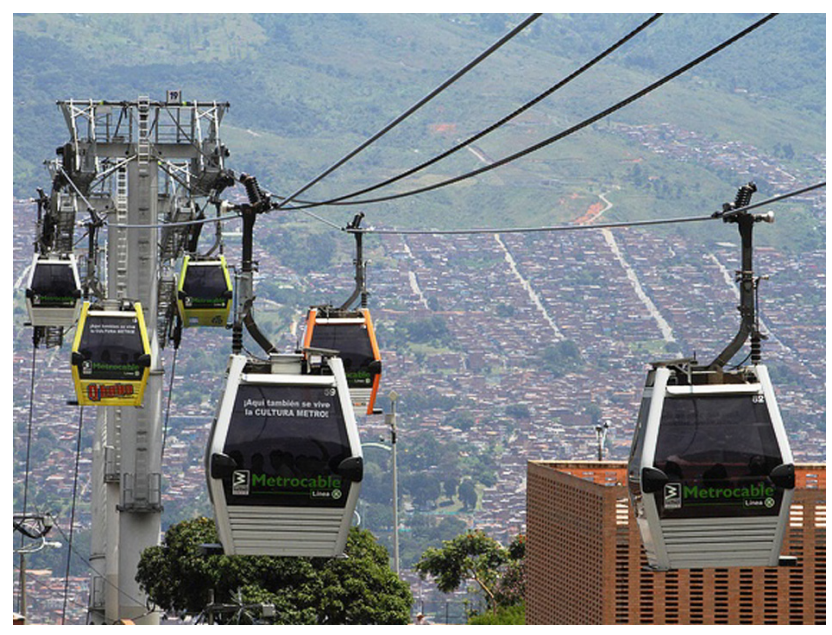

Figura 4. Sistema de Transporte por Cable Metrocable, Medellín, Colombia. Fuente: https://c1.staticflickr.com/5/ 4108/4968401847_b34d09491b_z.jpg

En cuanto al transporte individual, se encuentra la bicicleta, que en muchas ciudades alrededor del mundo ha dejado de ser un medio de transporte de uso particular y ha sido implementada como transporte público. La dinámica del sistema consiste básicamente en el alquiler de bicicletas a los usuarios para viajes cortos o, como medios alimentadores de sistemas masivos de transporte existentes como autobuses, ferrovías, entre otros; maximizando así la utilidad de estos últimos y minimizando la inversión en infraestructura para sistemas alimentadores. Según Kisner (2011) una de las tantas ventajas del uso masivo de bicicleta como transporte público, es la evidente baja en la congestión de vías, lo que conlleva a la disminución en el consumo de combustibles fósiles por parte del parque automotor y esto a su vez, desencadena en la disminución de emisiones de gases de efecto invernadero y la mejora en la calidad del aire, además de la innegable mejora en la salud de las personas debido a la actividad física realizada por el uso de la bicicleta. Un ejemplo de la implementación de este sistema de transporte urbano es la capital colombiana, Bogotá, que fue catalogada, en 2013, como una de las dieciocho mejores ciudades para andar en bicicleta pues cuenta con $344 \mathrm{Km}$ de carril para bicicleta con una buena cobertura del área urbana (Fernández, 2013); la gran infraestructura de la ciudad le permitió adelantar en 2014 proyectos como la apertura del Biocorredor El Virrey, el cual cuenta con estaciones autosostenibles en las que se brinda el servicio de préstamo de bicicletas compartidas; estaciones que tienen suministro eléctrico mediante paneles solares y un sistema de recolección de aguas lluvias para su uso en el aseo de las bicicletas (Prensa IDRD, 2014). En la Tabla 2 se muestran algunas cifras de los sistemas de bicicletas compartidas de algunas ciudades alrededor del mundo para 2014.

Por último, es necesario mencionar los sistemas peatonales, pues son parte fundamental de la movilidad urbana aunque en muchas ocasiones sean considerados como un componente de los sistemas vehiculares y no como un homólogo de estos. Caminar es el medio de desplazamiento básico y quizá el más económico y accesible a la población en general. Como todo sistema, los sistemas peatonales también tienen componentes entre los que están el Sistema de Movilidad (Campañas, Multas, Control del Tráfico, Movilidad vehicular y peatonal), la Infraestructura (Vías, Puentes Vehiculares y Peatonales, Señalización), las Políticas y Normatividad y el Presupuesto (Álvarez et ál., 2014).

Las principales ventajas de este sistema de transporte son cero emisiones de gases efecto de invernadero por uso de combustibles fósiles y la mejora en la calidad de vida de las poblaciones que optan por esta alternativa de transporte pues se someten a varios minutos e incluso horas de caminata al día; sin embargo es de suma importancia la organización y planeación de la infraestructura (Figura 5), teniendo en cuenta la seguridad vial en la interacción del peatón con los vehículos pues los transeúntes son muy susceptibles a tener accidentes que evidentemente pueden causar daños físicos, temporales o permanentes. Otros 
Tabla 2. Cifras de algunos de los sistemas de bicicletas compartidas del mundo.

\begin{tabular}{|c|c|c|c|c|c|c|c|c|c|c|c|c|c|c|c|c|c|c|}
\hline \multicolumn{5}{|c|}{ 1. Londres } & \multicolumn{4}{|c|}{ 2. París } & \multicolumn{4}{|c|}{ 3. Barcelona } & \multicolumn{6}{|c|}{ 4. Lyon } \\
\hline \multicolumn{5}{|c|}{ Reino Unido } & \multicolumn{4}{|c|}{ Francia } & \multicolumn{4}{|c|}{ España } & \multicolumn{6}{|c|}{ Francia } \\
\hline \multicolumn{5}{|c|}{ Bardays Cycle Hire } & \multicolumn{4}{|l|}{ Vélib' } & \multicolumn{4}{|l|}{ Bicing } & \multicolumn{6}{|l|}{ Vélo'v } \\
\hline \multicolumn{5}{|c|}{ Bicicletas: 7.000} & \multicolumn{4}{|c|}{ Bicicletas: 16.500} & \multicolumn{4}{|c|}{ Bicicletas: 4.100} & \multicolumn{6}{|c|}{ Bicicletas: 3.000} \\
\hline \multicolumn{5}{|c|}{ Estaciones: 554} & \multicolumn{4}{|c|}{ Estaciones: 1.751} & \multicolumn{4}{|c|}{ Estaciones: 420} & \multicolumn{6}{|c|}{ Estaciones: 347} \\
\hline \multicolumn{5}{|c|}{ Estacionamientos: 140.000} & \multicolumn{4}{|c|}{ Estacionamientos: 40.421} & \multicolumn{4}{|c|}{ Estacionamientos: 10.580} & \multicolumn{6}{|c|}{ Estacionamientos: 6.400} \\
\hline \multicolumn{5}{|c|}{$\begin{array}{l}\text { Costos de la Suscripción: } \\
\text { En dólares }\end{array}$} & $\begin{array}{l}\text { Costos } \\
\text { En dóla }\end{array}$ & $\begin{array}{l}\text { de la Suser } \\
\text { res }\end{array}$ & ripción: & & $\begin{array}{l}\text { Costos } \\
\text { En dóla }\end{array}$ & $\begin{array}{l}\text { de la Susc } \\
\text { ares }\end{array}$ & ripción: & & \begin{tabular}{|l|} 
Costos \\
En dóla \\
\end{tabular} & $\begin{array}{l}\text { de la: } \\
\text { res }\end{array}$ & uscri & pción: & & \\
\hline Anual & Mensual & Sem & Ianal & Diari & io Anual & Mensual & Semanal & Diario & Anual & Mensual & Semanal & Diario & Anual & Men & inal & Sema & nal & Diario \\
\hline 123 & $\mathrm{~N} / \mathrm{A}$ & 13 & & 3 & 38 & N/A & 11 & 2 & 62 & N/A & N/A & N/A & 33 & $\mathrm{~N} / \mathrm{A}$ & & 5 & & 2 \\
\hline Operado & or: Privado & & & & Operad & or: Privado & & & Operad & lor: Privad & & & Operad & or: Pri & ado & & & \\
\hline 5. Mont & treal & & & & & 6. Washin & ngton DC & & & & & 7. Guar & ngzhou & & & & & \\
\hline Canadá & & & & & & Estados Us & nidos & & & & & China & & & & & & \\
\hline Bixi & & & & & & Capital Bi & ikeshare & & & & & Guang & zhou Pu & blic Bi & ycle & & & \\
\hline Biciclet & as: 3.800 & & & & & Bicicletas: & $: 1.800$ & & & & & Biciclet & tas: 5.00 & & & & & \\
\hline Estacion & nes: 411 & & & & & Estaciones & s: 238 & & & & & Estacio & nes: 109 & & & & & \\
\hline Estacion & namientos: & 7.76 & & & & Estacionan & mientos: 3 . & 750 & & & & Estacio & namient & s: 2.2 & & & & \\
\hline $\begin{array}{l}\text { Costos } \\
\text { En dólat }\end{array}$ & $\begin{array}{l}\text { de la Suscr } \\
\text { res }\end{array}$ & ripcic & & & & $\begin{array}{l}\text { Costos de } \\
\text { En dólares }\end{array}$ & la Suscri & ción: & & & & $\begin{array}{l}\text { Costos } \\
\text { En dóla } \\
\end{array}$ & $\begin{array}{l}\text { de la Su } \\
\text { ares }\end{array}$ & scripc & & & & \\
\hline Anual & Mensua & & Semar & & Diario & Anual & Mens & & Semana & $\mathrm{Di}$ & ario & Anual & $\mathrm{Me}$ & ssual & Sem & tanal & $\mathrm{Di}$ & ario \\
\hline 80 & 30 & & N/A & & 7 & 75 & 25 & & N/A & 7 & & N/A & $\mathrm{N} / \mathrm{A}$ & & $\mathrm{N} / \mathrm{A}$ & & $\mathrm{N} /$ & \\
\hline Operado & or: Público & & & & & Operador: & Privado & & & & & Operad & or: Públi & & & & & \\
\hline 8. Hang & gzhou & & & & & 9. Shangh & & & & & & 10. Mé & xico DF & & & & & \\
\hline China & & & & & & China & & & & & & México & & & & & & \\
\hline Hangzh & 1ou Public & Bicy & & & & & & & & & & EcoBic & & & & & & \\
\hline Biciclet & as: 66.500 & & & & & Bicicletas: & $: 28.000$ & & & & & Biciclet & tas: 3.20 & & & & & \\
\hline Estacion & nes: 2.700 & & & & & Estaciones & s: 330 & & & & & Estaciol & nes: 279 & & & & & \\
\hline Estacion & namientos: & N/A & & & & Estacionan & mientos: $\mathrm{N}$ & & & & & Estacio & namient & s: 7.1 & & & & \\
\hline $\begin{array}{l}\text { Costos } \\
\text { En dólat }\end{array}$ & $\begin{array}{l}\text { de la Suscr } \\
\text { res }\end{array}$ & ripcic & & & & $\begin{array}{l}\text { Costos de } \\
\text { En dólares }\end{array}$ & la Suscri] & ción: & & & & $\begin{array}{l}\text { Costos } \\
\text { En dóla } \\
\end{array}$ & $\begin{array}{l}\text { de la Su } \\
\text { ares }\end{array}$ & scripc & & & & \\
\hline Anual & Mensua & & Semar & & Diario & Anual & Mens & & Semana & $\mathrm{Di}$ & ario & Anual & $\mathrm{Me}$ & ssual & Sem & ranal & $\mathrm{Di}$ & ario \\
\hline $\mathrm{N} / \mathrm{A}$ & N/A & & N/A & & N/A & N/A & N/A & & N/A & $\mathrm{N} /$ & & 31 & $\mathrm{~N} / \mathrm{A}$ & & 24 & & 7 & \\
\hline Operadc & or: Público & & & & & Operador: & Público & & & & & Operad & or: Priva & & & & & \\
\hline
\end{tabular}

Fuente: Adaptado de http://www.elespectador.com/files/imagecache/560_width_display/img_ipad/339b2260867db6770af550551a6fa569.jpg

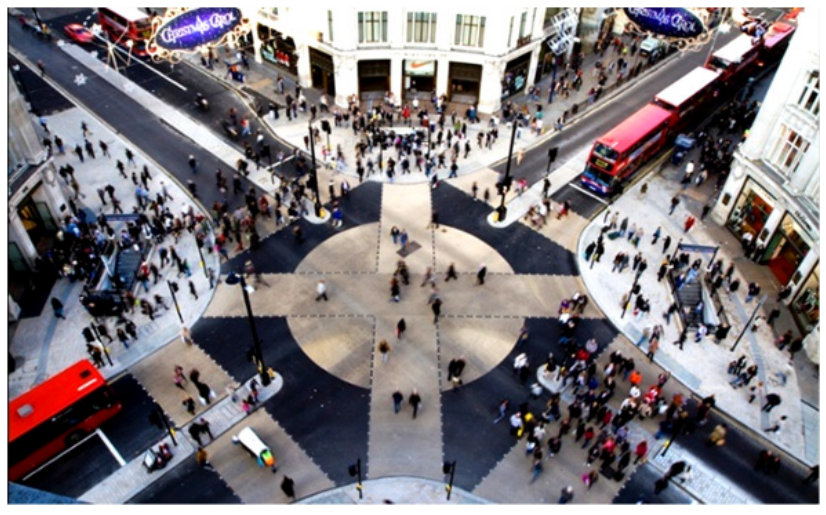

Figura 5. Peatones en el cruce de carreteras en diagonal en Oxford Circus en Londres. Fuente: Félix Arcilla. http:// i.guim.co.uk/static/w-620/h—/q-95/sys-images/Guardian/ Pix/pictures/2014/7/7/1404729213648/cdf451dd-418e-4560ac5a-a141f2c193e2-620x372.jpeg beneficios colaterales son la disminución del uso del vehículo particular y con ello la descongestión de corredores viales que se traduce en menos emisiones y al haber más gente transitando en las calles se dan más interacciones sociales en los diferentes establecimientos y lugares comerciales de la ciudad favoreciendo la economía de vendedores y comerciantes (International Transport Forum, 2011).

\section{Conclusiones}

La problemática de movilidad en áreas urbanas guarda una relación directa con el rápido crecimiento urbanístico de las ciudades y el desarrollo de asentamientos suburbanos en zonas periféricas, los cuales generan un elevado número de viajes y a la vez 
mayores problemas de movilidad como la congestión, entendida como el exceso de demanda de viajes sobre su oferta, que trae consigo impactos sobre el medio ambiente urbano como lo son el consumo energético de fuentes no renovables, el incremento en los niveles de contaminación atmosférica, emisiones de gases contaminantes y ruido; problemas característicos de los sistemas de transporte público colectivo que emplean buses que se desplazan en vías no exclusivas, $\mathrm{y}$ el incremento del parque automotor de vehículos particulares en las ciudades.

Modelos de transporte masivo como el metro y el Bus Rapid Transit (BRT) se muestran como los primeros sistemas en considerar dentro de los esquemas de operación de alto rendimiento en términos de mayores cantidades de viajes así como la disminución de los tiempos de recorrido y las demoras ocasionadas por la congestión del tráfico, a la vez que buscan la disminución de los índices de accidentalidad y garantizar la seguridad y comodidad de sus usuarios. Estos sistemas ofrecen beneficios adicionales como la disminución de los impactos en el medio ambiente y por consiguiente los índices de contaminación ambientaly consumo energético.

Los sistemas de transporte de cero consumo de combustibles fósiles (tranvías, sistemas por cables, bicicletas compartidas y peatones) son los que, simultáneamente, presentan más beneficios y ventajas a nivel social, ambiental y de movilidad, pues son de fácil acceso a la población en general y sobre todo a la de menos recursos económicos; al no necesitar combustibles de origen fósil no emiten gases efecto invernadero, son sistemas que disminuyen el uso del vehículo particular colaborando nuevamente con la disminución de emisiones y mejorando la movilidad de las ciudades.

Hablando específicamente de los sistemas de bicicletas compartidas y peatones, proporcionan la mejora de la salud física y mental de las personas ya que desarrollan actividad física. Estos dos sistemas son de bastante contacto e interacción de los usuarios con los sistemas vehiculares, entonces es importante tener en cuenta la seguridad vial en las zonas de circulación e interacción de los dos tipos de sistemas.

\section{Referencias bibliográficas}

Alam, M., Ahmed, F., 2013. Urban transport systems and congestion: a case study of indian cities. Transport and Communications Bulletin for Asia and the Pacific, 82, pp. 33-43.
Alcântara, E., 2010. Análisis de la movilidad urbana. Espacio, medio ambiente y equidad. Corporación Andina de Fomento, ISBN 978-980-6810-60-0, Bogotá, Colombia, pp. 22, 204.

Alstom, 2014. Citadis on the move. The tram solution for each city. Alstom, September 2014, Paris, France, pp. 1-16.

Álvarez, P.L., Méndez, G.G., Martins, G.N., 2014. Los Sistemas Peatonales como Sistemas de Transporte. 15 p. Consultado el 30 de Junio de 2015. http:// revistas.udistrital.edu.co/ojs/index.php/revcie/ article/viewFile/8456/9976

Arboleda, M.A., Parra, I.F., Aristizábal, I., Sabogal, H., 2012. Estudio dinámico de la movilidad en la ciudad de Santiago de Cali - Colombia. X Congreso Latinoamericano de Dinámica de Sistemas, III Congreso Brasileño de Dinámica de Sistemas, I Congreso Argentino de Dinámica de Sistemas, $10 \mathrm{p}$. Consultado el 18 de Junio de 2015. http://www. dinamica-de-sistemas.com/revista/dinamica-de-sistemas-17.pdf

Banco de Desarrollo de América Latina CAF, 2011. Desarrollo urbano y movilidad en América Latina. Dirección de Análisis y Programación, Sectorial de la Vicepresidencia de Infraestructura de Corporación Andina de Fomento, Panamá, 317 p.

Banco Mundial, 2002. Ciudades en movimiento. Revisión de la estrategia de transporte urbano del Banco Mundial. Noviembre 2002, 253 p.

Centro Conjunto de Investigación sobre el Transporte de la OCDE y el Foro Internacional del Transporte, 2011. International Transport Forum. Peatones: seguridad vial, espacio urbano y salud. Informe de investigación Resumen. (C) OECD/ITF 2011, 24 p. Consultado el 30 de Junio de 2015. http://www. internationaltransportforum.org/pub/pdf/11Pe destrianSumES.pdf

Centre d'études sur les Réseaux, les Transports, l'urbanisme et les Constructions Publiques CERTU, 2011. Aerial cableways as urban transport systems. Ministère de l'Ecologie, du Développement durable, des Transports et du Logement. p. 3.

Colmenares, I.J., 2007. Desarrollo sustentable y sostenible de sistemas de transporte público urbano: impacto en la gerencia, organización y liderazgo. Seminario “Administración: Teorías y Categorías 
de Análisis", Universidad Central de Venezuela, Caracas, Venezuela, 1, $14 \mathrm{p}$.

Dangond Gibsone, C., Jolly, J.-F., Monteoliva Vilches, A., Rojas Parra, F., 2011. Algunas reflexiones sobre la movilidad urbana en Colombia desde la perspectiva del desarrollo humano. Papel Político, Vol. 16, No. 2, julio-diciembre, p. 485-514.

Environmental Protection Agency EPA, 2011. Guide to sustainable transportation performance measures. EPA 231-K-10-004. Southwestern Pennsylvania Commission (in Pittsburgh, Pennsylvania) and the Mid-America Regional Council (in Kansas City, Missouri), 3, $55 \mathrm{p}$.

Fedesarrollo, 2013. La integración de los sistemas de transporte urbano en Colombia: una reforma en transición. Centro de Investigación Económica y Social, Fedesarrollo, Agosto 31 de 2013, 112 p.

Fernández, M. A., 21 de Febrero de 2013. Bogotá entre las 18 mejores ciudades del mundo para ir en bicicleta. Consultado el 30 de Junio de 2015. http:/ /www.bogota.gov.co/article/bogot\%C3\%A1-entre-las-18-mejores-ciudades-del-mundo-para-iren-bicicleta.

Griffiths, P., 2012. Technology Briefing Paper Catenary Free Tram Operation. United Kingdom Tram. pp. $1-37$.

González, C.A., 2011. Proyectos de transporte e infraestructura y su contribución a la movilidad sostenible y la gobernabilidad local: el caso de Cali, Colombia. Revista Entorno Geográfico, No. 7-8, Enero/Diciembre 2011, Departamento de Geografía, Universidad del Valle, Cali, Colombia, p. 24-45.

Holuigue, C., 2011. Institucionalidad y transporte público urbano: Santiago de Chile y Medellín, Colombia. Proyecto Innovación ambiental de servicios urbanos y de infraestructura: Hacia una economía baja en carbono. Comisión Económica para América Latina y el Caribe (CEPAL), Impreso en Naciones Unidas, Santiago de Chile, Chile, 68 p.

Jirón, P., 2012. La importancia de la experiencia de movilidad en la planificación del transporte. Aprendizajes de Santiago de Chile. Movilidad Urbana \& Pobreza. Aprendizajes de Medellín y Soacha, Colombia. The Development Planning Unit UCL, Universidad Nacional de Colombia Sede Medellín. p. 23-30.
Kisner, C., 2011. Integrating Bike Share Programs into a Sustainable Transportation System. A product of the National League of Cities, in conjunction with its Sustainability Partner, The Home Depot Foundation, $4 \mathrm{p}$.

Landrin, S., 2014. The Guardian. Lift-off for urban cable car projects as cities seek transport solutions: France is the latest country to master the air and allow pedestrians to rise above the jams. Tuesday 6 November 2012. Consultado el 18 de Junio de 2015. http://www.theguardian.com/world/2012/ nov/06/cable-cars-transport-solutions-france

Lange Valdés, C., 2011. Dimensiones culturales de la movilidad urbana. Revista Invi, Vol. 26, No. 71, May 2011, p. 87-106.

Lara, J., Moreno, G., 2014. Movilidad urbana en Caracas. Un enfoque desde las tecnologías limpias para la formación de competencias ciudadanas para el desarrollo sostenible. Provincia, No. 31, enero-junio, pp. 11-48.

Leibler, L., Brand, P., 2012. Movilidad e inclusión social: la experiencia desde la periferia de Medellín y el primer Metrocable. Bulletin de l'Institut Français d'Études Andines, 41 (3), pp. 364-387.

Litman, T., Burwell, D., 2006. Issues in sustainable transportation. Int. J. Global Environmental Issues, 6(4), pp. 331-347.

Lizárraga Mollinedo, C., 2006. Movilidad urbana sostenible: un reto para las ciudades del siglo XXI. Economía, Sociedad y Territorio, septiembrediciembre, VI(22), pp. 1-35.

London Assembly Transport Committee, 2004. Tram, trolley or guided bus: what are the best choices for London? Proceedings of a seminar held by the Transport Committee. Published by Greater London Authority, City Hall, The Queen's Walk, London, April 2004, 54 p.

Lupano, J.A., Sánchez, R.J., 2009. Políticas de movilidad urbana e infraestructura urbana del transporte. Comisión Económica para América Latina y el Caribe CEPAL. Organización de Naciones Unidas ONU. Santiago de Chile, Chile. 65 p.

Mesquita Moreira, M., Volpe, C., Blyde, J.S., 2008. Unclogging the Arteries. The Impact of Transport Costs on Latin American and Caribbean Trade. Special Report on Integration and Trade Inter- 
American Development Bank David Rockefeller Center for Latin American Studies, Harvard University, Washington DC, United States, 179 p.

Ministry for Ecology, Sustainable Development and Energy, 2012. The tramway revival in France. Ministère de l'Écologie, du Développement durable et de l'Énergie, Secrétariat général, May 2012, p.10.

Navas, A., 2008. Políticas de transporte público urbano: lecciones desde la experiencia de Transantiago. Pap. Polít. Estud., Vol. 4, No. 1, p. 159-189, enero-junio de 2008, Bogotá D.C., Colombia.

Orro, A., Novales, M., Rodríguez, M., 2003. Transporte por cable. Cuadernos del Grupo de Ferrocarriles y Transportes. Escuela Superior de Ingenieros de Caminos, Canales y Puertos, Universidad de la Coruña, Editorial Tórculo Artes Gráficas, A Coruña, Septiembre de 2003, 144 p.

Prensa IDRD, 27 de Junio de 2014. Con gran éxito se puso al servicio Bicicorredor del Virrey. Consultado el 30 de Junio de 2015. https://www.youtube. $\mathrm{com} /$ watch? $\mathrm{v}=$ UhBoyvWoz8Q\&feature=youtu.be
Rao, A.M., Rao, K.R., 2012. Measuring urban traffic congestion - a review. International Journal for Traffic and Transport Engineering, 2012, 2(4): pp. 286-305.

República de Colombia. Ministerio de Ambiente, Vivienda y Desarrollo Territorial, 2006. Ley 1083 del 31 de Julio de 2006: por medio de la cual se establecen algunas normas sobre planeación urbana sostenible y se dictan otras disposiciones. Bogotá D.C., Colombia, 5 p.

Transportation Research Board, 2005. Critical issues in transportation. Transportation Research Board, Of The National Academies, Washington DC, United States, $16 \mathrm{p}$.

World Economic Forum, 2012. Sustainable Transportation Ecosystem. Addressing sustainability from an integrated systems perspective. April 2012, Cologny, Geneva, Switzerland, 56 p. 
